\title{
Effects of Methadone Maintenance Therapy on Thyroid Function of Adult Men
}

\author{
Shahrzad Bozchelou' and Mohammad Delirrad ${ }^{2}$ \\ ${ }^{1}$ Toxicology Department, Islamic Azad University, Ahar, Iran \\ ${ }^{2}$ Department of Forensic Medicine and Clinical Toxicology, University of Medical Sciences, Urmia, Iran
}

\begin{abstract}
One of the major challenges in methadone maintenance therapy (MMT) for drug dependence is the physiological side effects on endocrine hormones. Because of the key role of the thyroid gland in the normal functioning of the human body and brain, this study examined the effect of MMT on thyroid function. Thyroid hormones (T3, T4, and thyroid-stimulating hormone (TSH)) were evaluated in normal and user treated with MMT who were referred to the Province Clinical \& Pathology Center of Urmia, Iran. The study was conducted for three months using the Case Series method. A total of 270 samples were collected, 215 were from individuals who were not treated, whereas 55 were from men treated with methadone. Average levels of T3 and T4 in non-treated sample of men are $1.34 \pm 0.02 \mathrm{ng} / \mathrm{mL}$ and $90.96 \pm 1.38 \mathrm{ng} / \mathrm{mL}$ while the corresponding values for patients treated with methadone are $1.39 \pm 0.04 \mathrm{ng} / \mathrm{mL}$ for T3 and $94.57 \pm 2.72 \mathrm{ng} / \mathrm{mL}$ for T4. Mean TSH levels of the non-treated group and the methadone consuming group were $1.75 \pm 0.08 \mu \mathrm{IU} / \mathrm{mL}$ and $3.17 \pm 0.45 \mu \mathrm{IU} / \mathrm{mL}$, respectively. These results indicate that although men treated with methadone had higher levels of T3, T4, and TSH than normal individuals, only the difference in TSH level was significant. The importance of this difference among individuals on methadone maintenance programs should be investigated in larger samples over long periods of time. Additionally, the effects of methadone treatment on women should be examined.
\end{abstract}

Key words: Methadone maintenance treatment, Radioimmunoassay, Thyroid hormones

\section{INTRODUCTION}

In underdeveloped countries and the third world, particularly in western Asia, the rate of heroin addiction is continuously increasing. One of the main reasons for using heroin and its derivatives, particularly opium, is the perception of the beneficial effects of such drugs against various diseases. Studies have been conducted to examine the effects of opium addiction as well as the effects of various opiate addiction therapies such as methadone maintenance therapy (MMT) on various systems and factors such

Correspondence to: Shahrzad Bozchelou, The Province Clinical \& Pathology Center of Urmia, Kashani Street, Urmia, West Azerbaijan, 5715665896, Iran

E-mail: shahrzad.boozchaloo@gmail.com

This is an Open-Access article distributed under the terms of the Creative Commons Attribution Non-Commercial License (http:// creativecommons.org/licenses/by-nc/3.0) which permits unrestricted non-commercial use, distribution, and reproduction in any medium, provided the original work is properly cited. as endocrine hormones in the human body and laboratory animals (1-3).

Because endocrine hormones directly affect metabolism in the body, abnormalities in various hormones can occur during treatment, greatly affecting the efficacy of therapy. Therefore, determining the effects of these therapies on hormones may be useful for improving therapeutic methods and accurately interpret their effects.

Because of the increasing incidence and prevalence of drug abuse among youth and the rapid progress towards injection, comprehensive plans for treating substance abuse and its related conditions are essential. MMT is widely implemented by many national drug prevention programs to treat opioid drug use and prevent its harmful consequences to the individual user, his or her family, and society (4).

Methadone, a long-acting opioid agonist with a half-life of approximately $24-36 \mathrm{hr}$, was first synthesized as an anesthesia in Germany during the Second World War (5). In the 1950s and 1960s, Isbell and Vogel, who operated 
programs at the U.S. Public Health Hospital in Lexington, Kentucky and Riverside Hospital in New York City, showed that methadone is effective for treating heroin addiction (6). Since then, many national drug treatment programs have used methadone maintenance as a major strategy for treating drug users and preventing the negative effects associated with drug abuse. Among these countries is Iran, which has faced a massive problem of opioid drug abuse since the 1970s. Although methadone use is known cause changes in thyroid function, the precise mechanism remains unclear. Thus, we conducted a preliminary study in the Provincial Clinical \& Pathology Center of Urmia, Iran to investigate the impact of methadone use on the thyroid hormones T3, T4, and thyroid-stimulating hormone (TSH). The aim of this study was to determine whether the levels of these hormones differed in subjects addicted to opioids and using methadone compared to those in subjects not using methadone.

\section{MATERIALS AND METHODS}

Before collecting samples, the sample size, which indicates the number of samples required for statistical surveys, was determined. Because a similar study of humans has not been conducted, a pilot study was initially conducted to examine 21 healthy male individuals (control group) and 21 subjects who were drug-addicted and being administered methadone (case group) referred to the Provincial Clinical \& Pathology Center of Urmia. The levels of T3, T4, and TSH were measured in the two groups. Based on the results of this measurement, the sample size was calculated for determining the outcome of pre-treatment endometrial thickness using the following formula:

$$
\frac{z^{2} \times p \times(1-p)}{(0.03)^{2}}
$$

with $p=0.1$ considered as thyroid hormone levels in healthy adult male and addicted methadone recipients referred to the Provincial Clinical \& Pathology Center of Urmia. The confidence level of this test was $1-\alpha=95 \%$ and test power was $98 \%$. By replacing these values in the formula, the sample size excluding removed samples was found to be 270 individuals. The sampling method in this study was the total number to reach the desired sample size.

Primary screening was performed based on forms completed by the referrals, and the samples were based on methadone recipient addicts. Healthy male subjects with the fewest possible interventional factors based on declarations of respondent forms were selected and categorized. In the second stage, sample preparation was carried out as follows.

First, $5 \mathrm{cc}$ of blood was obtained from the selected individuals and then centrifuged at $1,500 \mathrm{rpm}$ for $5 \mathrm{~min}$ to obtain the required serum. In the next step, a gamma counter method was used to measure accurately and reliably measure hormone levels. For this step, the separated serum was tested using specific kits (Human Total Thyroxine (T4) RIA Kit, Human Total Tri-iodothyronine (T3) RIA kit, and Human Thyroid Stimulating Hormone (TSH) IRMA Kit (PGI Co., Tehran, Iran).

The T4 and T3 measurement kit methods are based on immunological assays and competitive radioactivity. Thus, in this test, T3 and T4 in the human samples compete for binding to a rabbit polyclonal antibody (anti-T3 and anti-T4) on the cover (coated) with T3 and T4 on the tube labeled with iodine-125. After incubation, the tubes were evacuated and the activity in each tube was measured with a gamma counter. The activity is inversely proportional to the concentration of T3 and T4 in the samples. The IRMA TSH kit is a solid-phase sandwich immunoradiometric assay that uses polyclonal anti-human hTSH antibodies immobilized on plastic tubes as capture reagents and an iodinated monoclonal antibody as a tracer (7). Tested TSH samples were exposed to both antibodies. After incubation, the tubes were drained and rinsed to remove excess antibody attached to iodine-125. The activity in each tube was measured with a gamma counter. Activity was directly proportional to the concentration of TSH in the samples (7).

For statistical data analysis, the measured hormone levels in the two groups were analyzed separately using SPSS software (SPSS, Inc., Chicago, IL, USA) and the mean and standard deviation for each hormone in the two groups were separately calculated by the software. As the number of available samples exceeded the calculated sample size, we did not examine the normal distribution, and instead used a parametric method (independent sample $t$-test and analysis of variance).

\section{RESULTS}

Thyroid hormone test results. For statistical analysis, the mean age of the two groups (case and control group) was calculated. The mean age \pm SD in the case and control groups were $41.62 \pm 9.85$ and $43.61 \pm 9.67$ years, respectively; the age difference between the two groups was not significant. After reviewing the equality of variances using

Table 1. Results of statistical analysis of hormones T3, T4, TSH levels in the normal adult men (control group) and addicts receiving methadone (case group) and calculated probability (p-value)

\begin{tabular}{lccc}
\hline \hline \multirow{2}{*}{ Hormone } & \multicolumn{2}{c}{ Hormone level (Mean $\pm \mathrm{SD})$} & \\
\cline { 2 - 3 } & $\begin{array}{c}\text { Case group } \\
(\mathrm{n}=55)\end{array}$ & $\begin{array}{c}\text { Control group } \\
(\mathrm{n}=215)\end{array}$ & \\
& $1.34 \pm 0.02$ & $1.39 \pm 0.04$ & 0.279 \\
$\mathrm{~T} 3 /(\mathrm{ng} / \mathrm{mL})$ & $90.96 \pm 1.38$ & $94.57 \pm 2.72$ & 0.239 \\
$\mathrm{~T} 4 /(\mathrm{ng} / \mathrm{mL})$ & $1.75 \pm 0.08$ & $3.17 \pm 0.45$ & 0.003 \\
$\mathrm{TSH} /(\mu \mathrm{IU} / \mathrm{mL})$ & $1.75 \pm$ \\
\hline
\end{tabular}


Table 2. Statistical analysis report (ANOVA) of the effects of methadone dose on thyroid hormones

\begin{tabular}{clrrrc}
\hline \hline Hormone & Compare group & Sum of squares & $\mathrm{d}_{\mathrm{f}}$ & $\mathrm{F}$ & $p$-Value \\
\hline \multirow{3}{*}{ TSH } & Between groups & 9.441 & 2 & 0.414 & 0.663 \\
& Within groups & 593.466 & 52 & & \\
& Total & 602.906 & 54 & & 0.553 \\
& Between groups & 0.128 & 2 & 0.599 & 0.651 \\
\hline \multirow{2}{*}{$\mathrm{T} 3$} & Within groups & 5.534 & 52 & & \\
& Total & 5.662 & 54 & & \\
& Between groups & 358.898 & 2 & & \\
\hline
\end{tabular}

Leven's test, independent sample $t$-test conducted to compare TSH, T3, and T4 levels in the two groups (Table 1). As shown in Table 1, the average T3, T4, and TSH levels in the case group were higher than those in the control group, but the difference was significant only for TSH.

Effect of methadone dose on thyroid hormones. To evaluate the effect of methadone dosage on thyroid hormone function in men treated with MMT, the case group was divided into groups based on the dose. The first group was treated with less than $100 \mathrm{mg}$ of methadone per day (35 samples), the second group people was administered $100-150 \mathrm{mg}$ of methadone per day (11 samples), and the third group was administered more than $150 \mathrm{mg}$ of methadone per day ( 9 samples). Examination of the hormone levels in these three groups by analysis of variance revealed no significant difference in T3, T4, and TSH levels. These results are summarized in Table 2.

\section{DISCUSSION}

Based on our results, MMT for treating opioid drug dependence significantly increases TSH levels in patients treated with methadone, but no meaningful changes were observed in T3 and T4 levels. This does not mean that changes in the level of these two hormones (T3 and T4) were not observed, but it means that, from a statistical analysis perspective, these changes in the level of T3 and T4 hormones do not have a scientific concept. We also evaluated the effect of the dose of methadone on the level of hormones in the blood serum. The results showed that differences in the dosage of methadone did not alter hormone levels in the patients.

There are contradictory reports regarding the impact of MMT on thyroid hormone levels (3,8-13). Our study revealed no significant changes in hormones $\mathrm{T} 3$ and $\mathrm{T} 4$ after treatment with methadone. These results agree with those reported by Schussler et al. (9); however, English et al. (10) reported contradictory results. According to his findings, the serum concentrations T3 and T4 were decreased by MMT.
There is no clear explanation for the different results of various studies. However, these differences may be related to the number of patients studied. Additionally, confounding factors in patients may cause variable results, as heroin addiction or abuse of other drugs can affect the analysis. Other factors may also cause abnormal protein binding of these hormones during measurement, and factors such as liver disorders, birth control pills, and abuse of other drugs may give different results. It is well-known that liver diseases significantly alter T3 and T4 concentrations (11).

The results of this study show that the concentrations of TSH in patients treated with methadone was significantly higher than that in healthy individuals, which agrees with the results of a previous study (10). Because TSH is secreted by the pituitary gland, these results are consistent with numerous reports demonstrating the hypothalamic-pituitary endocrine changes caused by drug dependence $(12,13)$. In contrast to our results, Vescovi et al. (3) found no significant changes in this hormone.

The presence of potential confounding factors may have affected the results when measuring the effects of the dose of methadone. However, our preliminary results indicated that the dose of methadone has no effect on the concentration of these hormones in the blood serum and do not affect thyroid function. Thus, methadone has no dosedependent effects on thyroid hormones.

\section{ACKNOWLEDGMENTS}

The author would like to thank the Province Clinical \& Pathology Center of Urmia for providing the necessary facilities to carry out the research and Professor Amir H. Mehryar and Dr. Reyhani Rad for interest in this work and helpful discussion.

\section{CONFLICT OF INTEREST}

The authors have no conflict of interest to disclose.

Received November 10, 2017; Revised June 6, 2018; Accepted August 9, 2018 


\section{REFERENCES}

1. Mami, S., Eghbali, M., Khosravi, A., Salati, A.P., Purmehdi Brojeni, M., Mami, F. and Hushmandfar, R. (2011) Effect of opium addiction on T4, T3 and TSH in male and female rats. Scientific Journal of Ilam University of Medical Sciences, 20, 17-22.

2. Bhoir, K.K., Suryawanshi, S.A. and Pandey, A.K. (2009) Effects of sub-lethal heroin administration on thyroid stimulating hormone (TSH), thyroid hormones (T3, T4) and thyroid gland of Musnorvegicus. J. Environ. Biol., 30, 989-994.

3. Vescovi, P.P., Gerra, G., Rastelli, G., Ceda, G.P. and Valenti, G. (1984) Effect of methadone on TSH and thyroid hormone secretion. Horm. Metab. Res., 16, 53-54.

4. Joseph, H., Stancliff, S. and Langrod, J. (2000) Methadone maintenance treatment (MMT): a review of historical and clinical issues. Mt. Sinai J. Med., 67, 347-367.

5. Bell, J. and Zador, D. (2000) A risk-benefit analysis of methadone maintenance treatment. Drug Safety, 22, 179-190.

6. Isbell, H. and Vogel, V.H. (1949) The addiction liability of methadone (amidone, dolphine, 10820) and its use in the treatment of the morphine abstinence syndrome. Am. J. Psych., 195, 909- 914.
7. Karir, T., Samuel, G., Sivaprasad, N. and Meera, V. (2005) Development of coated tubes RIA for serum T3 for production scale. J. Immunoassay Immunochem., 26, 77-87.

8. Kuhn, C.M. and Bartolome, M. (1984) Effect of chronic methadone administration on neuroendocrine function in developing rats. Dev. Pharmacol. Ther., 7, 384-397.

9. Schussler, G.C., Stimmel, B. and Korn, F. (1980) Increased serum thyroid hormone binding in narcotic addicts is due to liver disease. Am. J. Drug Alcohol Abuse, 7, 379-387.

10. English, T.N., Ruxton, D. and Eastman, C.J. (1988) Abnormalities in thyroid function associated with chronic therapy with methadone. Clin. Chem., 34, 2202-2204.

11. Carter, J.N., Eastman, C.J., Corcoran, J.M. and Lazarus, L. (1974) Effect of severe chronic illness on thyroid function. Lancet, 2, 971-974.

12. Kaptein, E.M. (1997) Hormone-specific alterations of T4, T3, and reverse T3 metabolism with recent ethanol abstinence in humans. Am. J. Physiol., 272, 191-200.

13. Benavides, M., Laorden, M.L., Garcia-Borron, J.C. and Milanes, M.V. (2003) Regulation of tyrosine hydroxylase PVN and medulla oblongata catecholaminergic cell groups innervating the PVN. Eur. J. Neurosci., 7, 103-112. 